\title{
Pioneros de un Siglo de Enseñanza Anatómica en la Ciudad de Concepción, Chile
}

\author{
Pioneers of a Century of Anatomical Teaching in the City of Concepción, Chile
}

Osorio, H. ${ }^{1}$; Toro, J. C. ${ }^{1}$; Schorwer, K. ${ }^{1}$; Riveros, A. ${ }^{2,3}$ \& Cardenas, J. ${ }^{4}$

OSORIO, H.; TORO, J.C.; SCHORWER, K.; RIVEROS, A. \& CARDENAS, J. Pioneros de un siglo de enseñanza anatómica en la ciudad de Concepción, Chile. Int. J. Morphol., 38(3):650-658, 2020.

RESUMEN: Revisando un siglo de historia, el desarrollo de las actividades anatómicas en la ciudad de Concepción, en el sur de Chile, no estuvieron ajenas a dificultades. El presente trabajo reunió los antecedentes históricos que relatan los inicios y desarrollo de la enseñanza de la anatomía en la naciente Universidad de Concepción. Se realizó una búsqueda que incluyó la revisión de libros históricos, revistas, archivos digitales y registros institucionales como actas y archivos fotográficos. La fundación de la Universidad, los inicios de las actividades anatómicas en 1919 y el aporte de sus principales pioneros, evidencian las dificultades de la enseñanza de esta disciplina en Chile. Desde una perspectiva histórica, conocer dentro de este siglo de enseñanza los recursos físicos utilizados para la disección cadavérica, el detalle de las metodologías pedagógicas utilizadas y los recursos anatómicos disponibles, resultan ser un aporte al conocimiento del desarrollo de la anatomía en Chile.

PALABRAS CLAVE: Anatomía; Concepción; Historia de la Anatomía; Enseñanza de la Anatomía; Monumento Nacional.

\section{INTRODUCCIÓN}

Concepción demanda una Universidad. "Sin verdad y esfuerzo no hay progreso" (Wilhelm, 1966), esta frase escrita en el hall de acceso al segundo piso del recinto que recibió en el año 1924 al primer año de Medicina de la Universidad de Concepción, ubicado en la calle O`Higgins 850, representa las dificultades que enfrentó la enseñanza de la anatomía en el sur de Chile.

El anhelo de desarrollar una formación universitaria en la ciudad de Concepción comenzó en el siglo XVIII. En esta ciudad, ubicada a unos 500 kilómetros al sur de Santiago, la orden de los jesuitas fundó al alero del Obispado de Concepción la Universidad Pencopolitana. Esta institución que funcionó entre 1724 y 1767, tuvo la aspiración de desarrollar una Escuela de Medicina, la cual se vio truncada por el violento terremoto y maremoto de 1751. En dicha catástrofe la ciudad tuvo que trasladar su emplazamiento, desde el actual Penco hacia el valle de la Mocha, ubicado entre los ríos Biobio y Andalien. A lo anterior se sumó la expulsión de los jesuitas en 1767, lo que finalmente postergó la creación de esta Escuela (Wilhelm, 1962; Cavieres, 2013).
Iniciado el siglo XX, la ciudadanía de la pujante Concepción demandaba la creación de una Universidad. Dicha iniciativa contó con la incansable labor de un comité que abogaba por la creación conjunta de tres pilares vitales, una Universidad, un Hospital Clínico y una Facultad de Medicina. Esta última se debía relacionar fuertemente con dicho Hospital. La fundación de estas instituciones, no exento de dificultades, fueron alcanzadas en diferentes momentos, siendo la primera de ellas, la creación de la Universidad de Concepción en 1919 (Muñoz, 1993).

Este comité estuvo conformado por un grupo de ilustres penquistas con una importante presencia masónica donde destacaron el Sr. Enrique Molina, Rector del Liceo de Hombres de Concepción y del Dr. Virginio Gómez, Director del Hospital San Juan de Dios (Da Costa, 1995).

\footnotetext{
** El Dr. Virginio Gómez nació en Los Ángeles en 1877, una vez recibido de médico cirujano obtuvo en 1904 una beca para perfeccionarse en Alemania (Luvel, 1968) (Fig. 1A). Posterior a un largo periplo que incluyó su participación en la primera guerra mundial, de regreso en Chile, en 1917 fue nombrado Director del Hospital San Juan de Dios de Concepción. En
}

\footnotetext{
${ }^{1}$ Departamento de Anatomía Normal y Medicina Legal. Facultad de Medicina, Universidad de Concepción, Concepción, Chile.

${ }^{2}$ Departamento de Ciencias Morfológicas, Facultad de Medicina y Ciencia, Universidad San Sebastián, Lientur 1457, Concepción 4080871, Chile.

${ }^{3}$ Programa de Doctorado en Ciencias Morfológicas, Universidad de La Frontera, Temuco, Chile.

${ }^{4}$ Departamento de Anatomía y Medicina Legal, Facultad de Medicina Universidad de Chile, Santiago, Chile.
} 

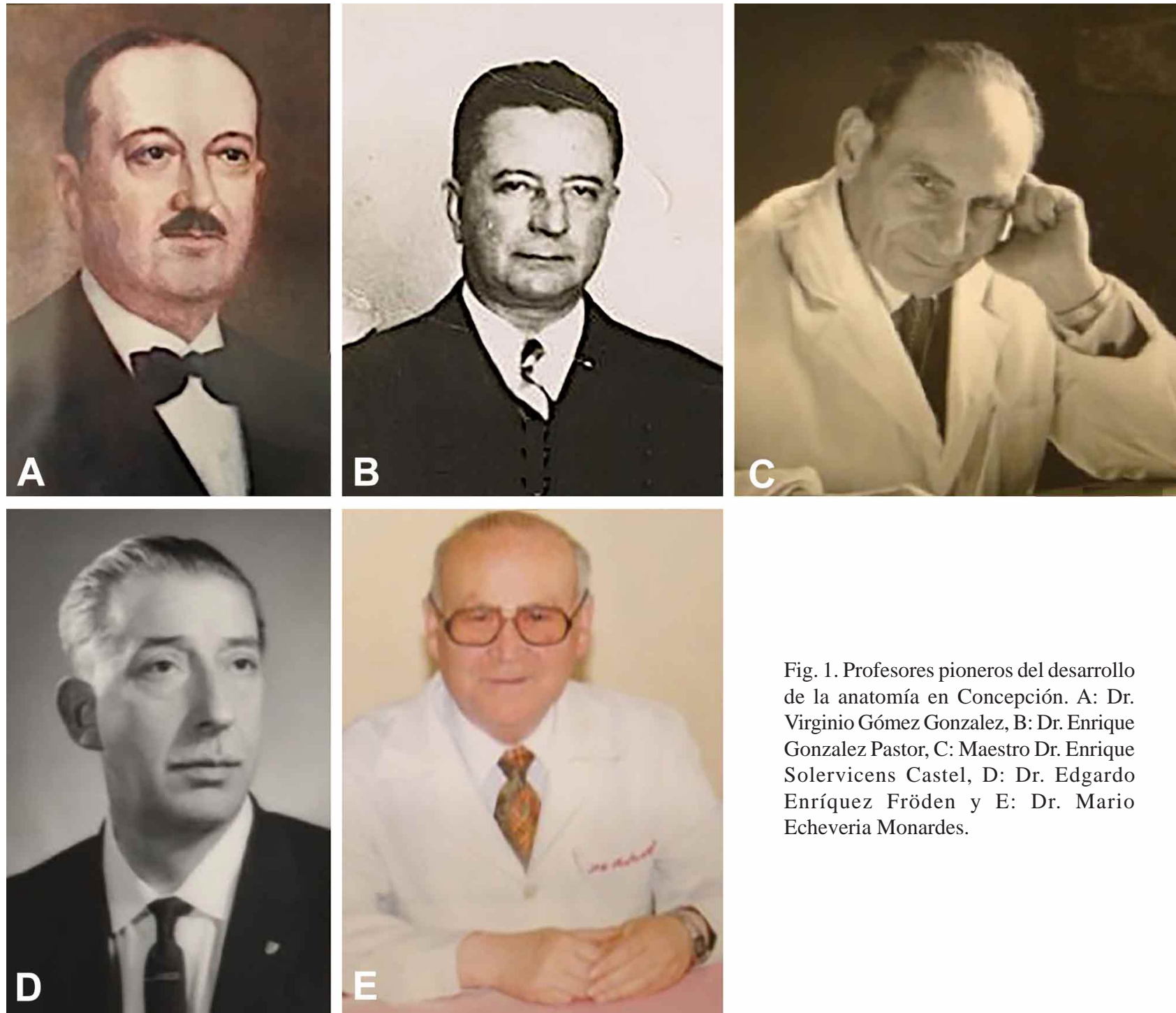

Fig. 1. Profesores pioneros del desarrollo de la anatomía en Concepción. A: Dr. Virginio Gómez Gonzalez, B: Dr. Enrique Gonzalez Pastor, C: Maestro Dr. Enrique Solervicens Castel, D: Dr. Edgardo Enríquez Fröden y E: Dr. Mario Echeveria Monardes.

este contexto desarrolló vínculos con la industria carbonífera, allí pudo constatar las lamentables condiciones sanitarias de la zona (Muñoz, 2015). Considerando que dichas condiciones serían muy perjudiciales para el desarrollo de la región, el Dr. Gómez manifestó que era indispensable para la ciudad, contar con un Hospital Clínico modelo, el cual fuera atendido por profesionales formados en la zona sur. A la luz de los posteriores resultados, el Hospital tuvo las mayores dificultades. Finalmente, el terremoto que azotó la región en 1939 destruyó el Hospital San Juan de Dios, lo que permitió la creación del Hospital Clínico (Da Costa, 1995).**

El trabajo del comité pro Universidad fue arduo; comenzó formalmente en marzo de 1917; recibió el apoyo de la comunidad que se tradujo en aportes filantrópicos de personas e instituciones de la zona sur (Da Costa, 2000). Finalmente y a pesar de innumerables gestiones realizadas por el Sr. Molina para que la naciente universidad fuera creada como institución estatal, el 17 de marzo de 1919, y realizando labores de presidente subrogante del comité, el
Dr. Gómez dio inicio a las actividades de la primera Universidad privada del país (Muñoz, 1993). Este hecho permitió concretar la aspiración de la comunidad de Concepción y de la zona sur de Chile de contar con una universidad para que sus hijos no tuvieran que viajar a Santiago para realizar sus estudios.

Primer Curso de Anatomía. En su primer año, la Universidad de Concepción impartió los primeros cursos de las carreras de Dentística, Química Industrial, Farmacia y Pedagogía en Inglés, además se sumó el curso de Leyes que ya se dictaba en el Liceo de Hombres. Correspondió al médico cirujano Dr. Virginio Gómez, ser el primer profesor de anatomía de la Universidad de Concepción. Aún cuando la Universidad comenzó a funcionar con 123 alumnos, de inmediato se estableció que uno de sus próximos objetivos prioritarios sería la creación de una 
Escuela de Medicina que cubriera la necesidad creciente de formar médicos cirujanos de excelencia, demanda que en ese entonces atendía con exclusividad la Universidad de Chile (Muñoz, 1993).

El curso de anatomía para Dentística, que tuvo una matrícula de 34 estudiantes, funcionó junto con las Escuelas de Farmacia y Química Industrial en un pequeño edificio de la calle O`Higgins 850 (Fig. 2A) (Da Costa, 2000). De los 34 estudiantes que completaron este curso, 29 aprobaron el examen final realizado por una comisión examinadora de profesores de la Universidad de Chile. Este curso teórico práctico incluía conferencias ilustradas con láminas dibujadas previamente a lo que se sumaban esquemas realizados por el docente en el pizarrón. El componente práctico se asociaba a demostraciones con material cadavérico y un número de disecciones guiadas por los ayudantes. Estas actividades se realizaban en una pequeña sala del Hospital San Juan de Dios, bajo la supervisión del Dr. Gómez y acompañado por los médicos cirujanos Nicanor Durán, Darío Pulgar, Enrique González Pastor y el dentista Víctor Villalobos (Solervicens, 1964). Al año siguiente, el Dr. Gómez dejaría el curso de anatomía en manos del Dr. Ladislao Labra Letelier, para asumir la cátedra de fisiología, la cual también dejó dos años más tarde. En 1924 el Dr. Gómez presentó su renuncia indeclinable al Directorio de la Universidad de Concepción, trasladándose a Valparaíso donde ejercería en la Compañía Sudamericana de Vapores. El año 1956, el Dr. Virginio Gómez falleció ahogado en el mar cuando viajaba a Valparaíso en el vapor Alondra (Muñoz, 2015).
En 1920, los nuevos estudiantes de Dentística realizaron sus pasos prácticos en un local ubicado en la calle Tucapel esquina San Martín (Fig. 2B). Los preparados cadavéricos debían ser trasladados desde el hospital en baldes, lo que dificultaba la realización de dichos trabajos; es por ello que previo a dejar sus labores en el Directorio de la Universidad, el Dr. Gómez gestionó los recursos para construir un pabellón de disección que estuviese anexo al Hospital San Juan de Dios, puntualmente en los terrenos cedidos por la Junta de Beneficencia de Concepción, en el barrio "La Toma” (Luvel; Muñoz, 1993).

Primer Pabellón de Anatomía. Iniciado el año 1921, el curso de anatomía para Dentística fue dictado por el Dr. Labra, el cual se realizó en una pequeña casa de madera frente al inicio de la calle Janequeo, esquina Chacabuco (Muñoz, 1993, García, 1994). El Dr. Solervicens la describiría: "contaba con dos salas, una grande de aproximados $20 \mathrm{~m}^{2}$ que se dispuso de dos mesas de cemento y tres o cuatro banquetas de madera. La segunda sala solo tenía $6 \mathrm{~m}^{2}$ y contaba con un lavatorio. El camino de acceso está formado por una hilera de piedra semi enterradas en el barro". Para llegar a este pabellón en los días lluviosos era menester de los profesores, ayudantes y estudiantes trasladarse desde la esquina de calle Janequeo con Cochrane por medio de carretones tirados por escuálidos caballos, luego de un pago de 20 centavos por persona (Luvel). Hay que recordar que la calle Janequeo estaba cubierta por adoquines sólo hasta la esquina de calle Cochrane, en la cual existía la única puerta de acceso al Hospital San Juan de Dios, y desde allí hasta el pabellón existía un lodazal. Este
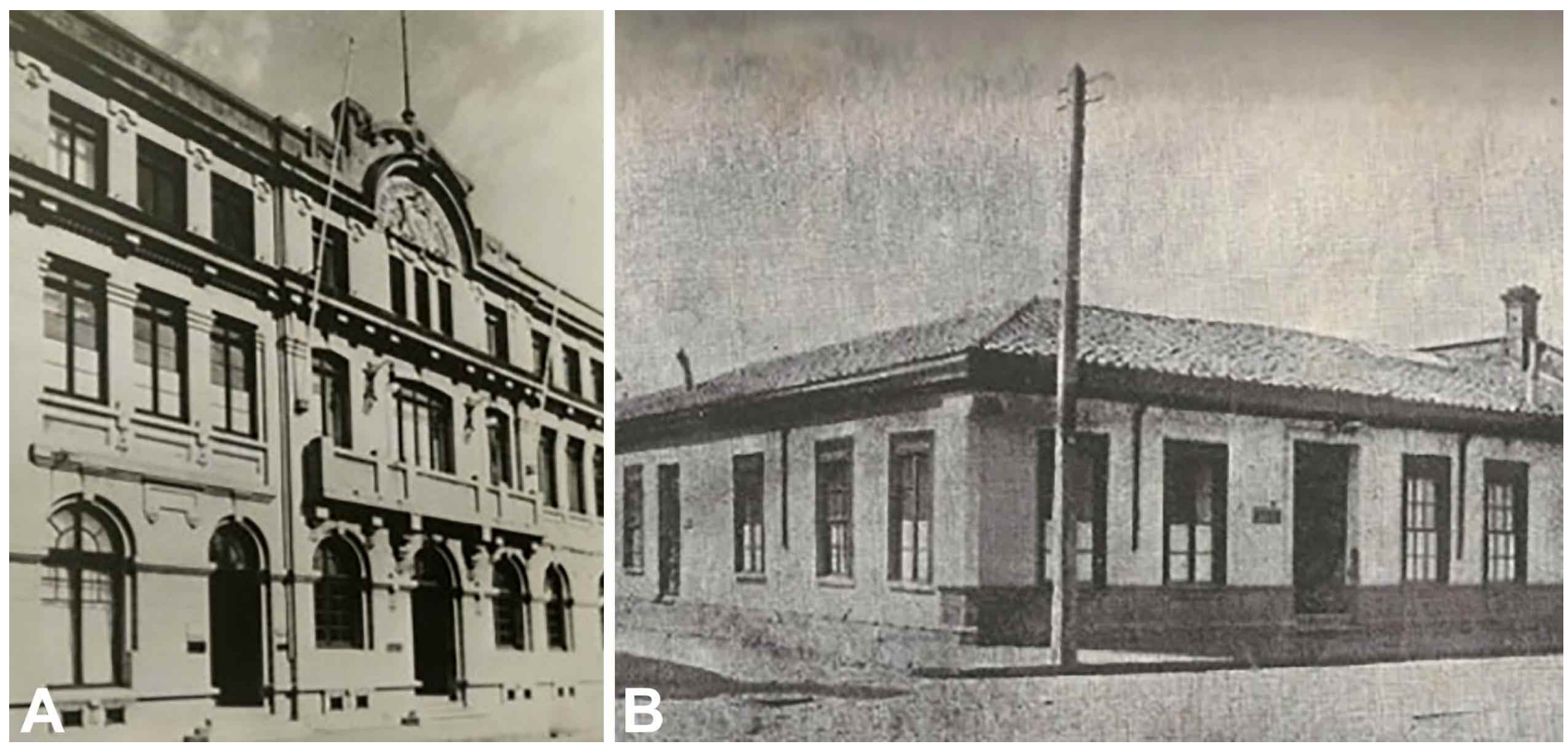

Fig. 2. Primeras instalaciones cursos de anatomía para Dentística. A: O`Higgins 850, B: Calle Tucapel esquina San Martín. 
pabellón funcionó unicamente para Dentística en los años 1922 y 1923, bajo la cátedra realizada por el Dr. Humberto Rojas Troncoso, acompañado de importantes ayudantes como Darío Pastene y Oscar Duarte. En el año 1924, este pabellón fue demolido y reemplazado por otro mas amplio que albergó los trabajos de disección del primer curso de anatomía para la naciente Escuela de Medicina (Solervicens; Muñoz, 1993).

Inicio Escuela de Medicina. En abril de 1924 se produce un hito importante para la docencia y desarrollo de la medicina chilena; efectivamente en Concepción, se creó el primer año de Medicina, comunicándose la noticia al país. Mientras en Concepción ocurría la inauguración de su Escuela, en Santiago las elevadas matrículas se cuadruplicaron en pocos años. Ejemplo de lo ello, es que en ese mismo año, el número estudiantes de primer año de Medicina en la Universidad de Chile era de 312, de los cuales 271 eran de reciente ingreso y 41 repitentes (Wilhelm, 1966; Jaramillo, 1924). Este hito presentó incluso mayores dificultades que la propia fundación de la Universidad de Concepción. Ya en 1921 el Rector de la Universidad Concepción, Sr. Enrique Molina envió un telegrama al entonces Decano de la Facultad de Medicina de la Universidad de Chile Dr. Gregorio Amunátegui, solicitando aprobación del proyecto de creación de los dos primeros años de la Escuela de Medicina en Concepción; dicha gestión no tuvo éxito. Recién a finales de 1923 y cuando en Santiago se seguía con las dificultades de la enseñanza generadas por el enorme número de estudiantes, el Dr. Amunátegui, ahora Rector de la Universidad de Chile y el Decano de la Facultad de Medicina Dr. Roberto Aguirre Luco dieron cuenta de las gestiones para autorizar que la Universidad Concepción dictara los primeros dos años de la Escuela de Medicina. El primero de estos dos años contó con los siguientes cursos: a) Botánica a cargo del Dr. Alcibíades Santa Cruz, b) Física por el Sr. Humberto Vergara, c) Química por el Sr. Salvador Gálvez y d) Zoología Médica por el Dr. Ottmar Wilhelm, en ese entonces pupilo del Dr. Juan Noé. El curso anatomía fue asignado al Dr. Enrique González Pastor (Wilhelm, 1966).

\footnotetext{
** El Dr. Enrique Gonzalez Pastor (Fig. 1B) nació en Concepción el 02 de enero de 1889. En 1913 se tituló con laureles de médico cirujano en la Universidad de Chile, realizando una tesis sobre la transplantación de órganos. Becado por el Gobierno de Chile, estuvo en París realizando estudios de urología y cirugía en general. Volviendo a Chile, se radicó en Concepción y formó parte activa del Comité Pro Universidad. Sus vínculos con anatomía fueron importantes pues en 1919 colaboró con el Dr. Gomez en el curso de anatomía para la carrera de Dentística, posteriormente, entre 1924 y 1927 dirigió el curso de anatomía para la recién creada Carrera de Medicina. En 1927 dejó la cátedra de anatomía para dictar el curso de Patología Quirúrgica, la cual realizó hasta su jubilación en 1945. Cabe destacar que durante su vida académica, el Dr. Gonzalez también desarrolló actividades investigativas donde destacaron sus monografías sobre dolor abdominal y fístulas vesicovaginales. Después de una larga lucha contra una enfermedad crónica el Dr. Gonzalez falleció en Septiembre de 1957
}

(Solervicens; Muñoz, 1993; Archivos del Departamento de Anatomía Normal y Medicina Legal Universidad de Concepción, 2019).

La inauguración de esta Escuela de Medicina contaría con una masiva concurrencia. En la mañana del 26 de abril de 1924 llegaron los invitados de todo el país, entre los que se encontraron autoridades como Pedro Aguirre Cerda, en ese entonces Senador por la provincia, Gregorio Amunátegui y Roberto Aguirre Luco, a los que se sumó, Edmundo Jaramillo, Director de la Escuela de Medicina de la Universidad de Chile acompañado por importantes profesores como Eugenio Díaz, Luis Vargas, Juan Noé y Carlos Charlín, entre otros (Wilhelm, 1966). Oficialmente las clases de la nueva Escuela de Medicina de la Universidad de Concepción se dieron por iniciadas con el comienzo del curso de Zoología Médica, a cargo del profesor Ottmar Wilhelm. Posteriormente, el Dr. Charlín haría un discurso de homenaje:" Pasteur, el hombre", como inauguración del curso de Medicina de Concepción, efectuado en el salón de actos del Liceo de Hombres. En la noche, durante el banquete de honor, el Dr. Aguirre Luco en un discurso señaló el júbilo y satisfacción que la inauguración de la nueva escuela provoca en la Facultad de Medicina de la Universidad de Chile: “... Saludo, en nombre de la Facultad de Medicina de Santiago, a los distinguidos colegas que van a tener la dirección de los cursos de medicina en Concepción; les conozco, ellos honrarán la cátedra y recordarán seguramente con gratitud y cariño a la madre común, a la vieja, a la querida Escuela de Medicina de la Universidad de Chile, que siente orgullosa de tener tales hijos ".

Por su parte, Salvador Gálvez, el primer Director de la Escuela de Medicina de la Universidad de Concepción pronució “... Hace cinco años, en forma modesta y silenciosa inauguraron sus clases los primeros cursos de las escuelas creadas por la Universidad de Concepción. Hoy, encontrándonos más fuertes, con más confianza en el porvenir, nos hemos permitido batir palmas, al anunciar el nacimiento de una escuela que era el anhelo de los dirigentes de la Universidad local y de la región".

Esta nueva Escuela de Medicina abrió considerando que los dos primeros años de la Carrera se ofrecerían en Concepción para luego rendir su Bachillerato en Santiago. Su primer curso, cuya enseñanza se impartía en conformidad a los programas de la Universidad de Chile, comenzó con 55 alumnos, entre ellos 4 mujeres. Al finalizar el año académico se presentaron a exámenes 38 estudiantes, aprobando el 42 $\%$, cifra porcentualmente superior a la de Santiago, aspecto a considerar, teniendo en cuenta que la comisión examinadora era de la Facultad de Medicina de la Universidad de Chile. Este curso de anatomía estuvo a cargo del Dr. Gonzalez acompañado de ayudantes entre los que destacaba un joven 
Enrique Solervicens, estudiante de medicina proveniente de Santiago y que cursaba su último año en el Hospital San Juan de Dios. Como este curso era bianual ocupaba un importante espacio de la carga horaria del primer y segundo año de la carrera (Muñoz, 1993). En el primer año se estudiaba el aparato locomotor y durante el segundo los restantes aparatos y sistemas. Este curso también consideraba actividades teóricas y prácticas. Las primeras consistieron en conferencias acompañadas de láminas, dibujos y preparaciones cadavéricas realizadas por los ayudantes. La parte práctica de este curso era controlada por los ayudantes y consistían en que cada alumno debía realizar un mínimo de 25 preparados cadavéricos por cada año de este curso (Solervicens).

Pabellón Veneciano. Iniciado el curso de anatomía para Medicina, el nuevo pabellón permitió el correcto desarrollo de estos cursos. El Dr. Solervicens lo describía así: "Este nuevo pabellón ofrecía ya ciertas comodidades de trabajo, pues, contaba con una sala de clases escalonada, una gran sala de disección, una salita de profesor, una salita de disección para los ayudantes, un depósito de concreto para conservar los cadáveres, instalaciones higiénicas y lavatorios" (Fig. 3). A pesar de las comodidades ya descritas, el profesor Dr. Gonzalez, bautizó este pabellón, como "Pabellón Veneciano". Dicho bautismo era evidencia del invierno en Concepción, ya que las lluvias ocasionaban que el predio quedara totalmente rodeado de agua, por lo que el profesor, su ayudante y los estudiantes tenían que ser rescatados por coches tirados por caballos. El agua inundaba el exterior del pabellón, subiendo hasta $60 \mathrm{~cm}$ pues existían dos canales abiertos; el que venía desde el parque Ecuador por calle Victor Lamas y el que corría por la calle Edmundo Larenas.

En 1927, el Directorio de la Universidad de Concepción aprobó la organización de las Escuelas en Facultades, siendo elegido como primer Decano el Dr. Alejandro Lipschütz, lo anterior no consideraba la interrupción de su relación con Universidad de Chile. A partir de ese año se inició un proceso de consolidación de la actividad académica por parte de la naciente unidad de anatomía. Hombre clave de este desarrollo fue el Dr. Enrique Solervicens Castel, quien, producto de la salida del Dr. Gonzalez, quedó a cargo del curso de anatomía para Medicina, sumándose el curso de anatomía para Dentística que ya realizaba desde 1924 (Muñoz, 1993).
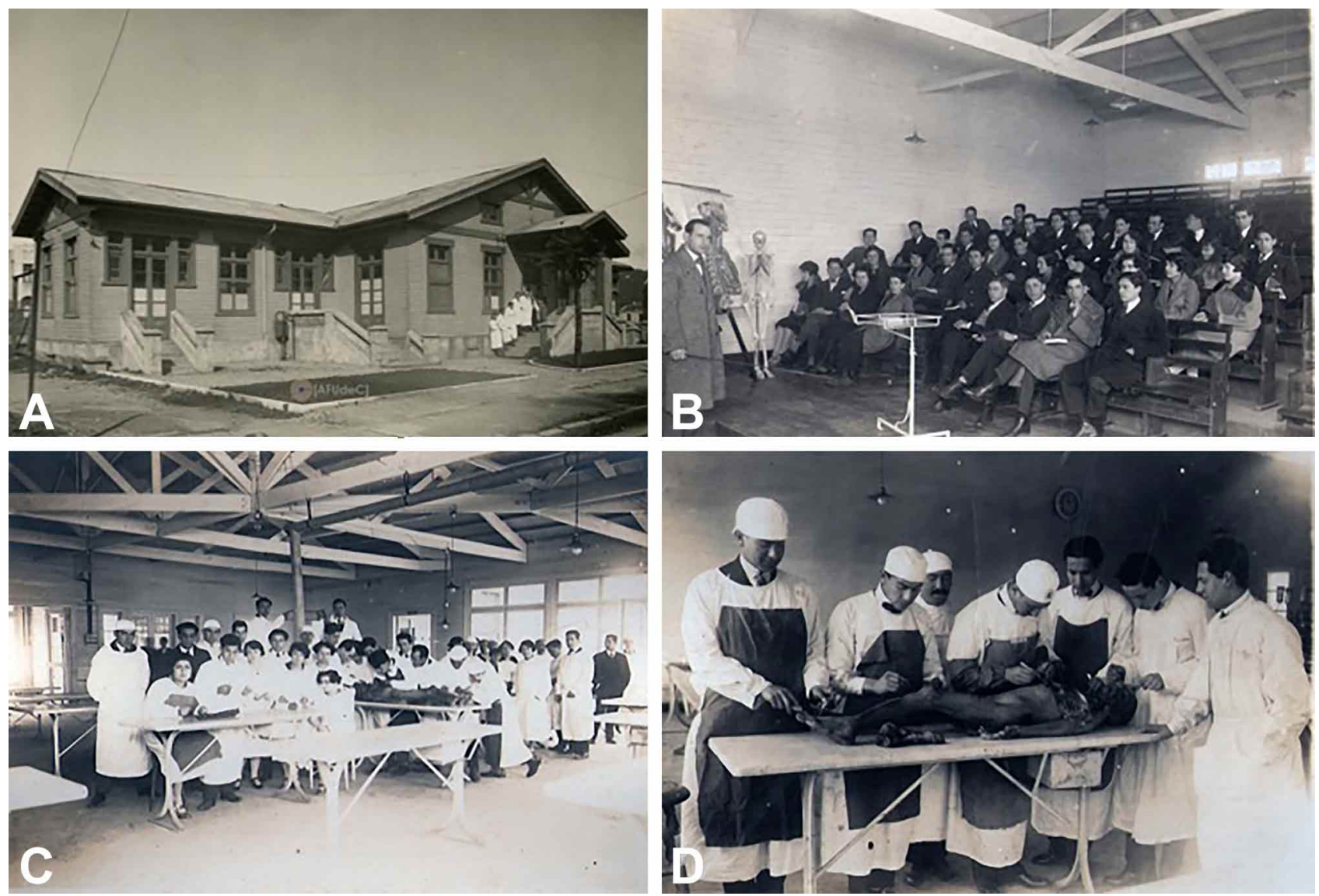

Fig. 3. Pabellón de Anatomía 1924 - 1933, bautizado como "Pabellón Veneciano". A: Fachada, B: Sala de clases, C: Sala de disección, D: Disección realizada por estudiantes y supervisada por ayudantes (Archivo fotográfico Universidad de Concepción AFUdeC). 
** El rol en el desarrollo de la anatomía por parte del Dr. Enrique Solervicens (Fig. 1C) es indiscutible, muestra de ello fue que el 3 de Septiembre de 1987, la Sociedad Chilena de Anatomía lo nombró de forma póstuma Maestro de la Anatomía Chilena (Cárdenas-Valenzuela \& Rodríguez-Torres, 2018). Nació en Mulchen el 17 de diciembre de 1899. Estudió Medicina en la Universidad de Chile, de la cual egresó el 8 de junio de 1925. Durante su tránsito estudiantil, fue discípulo del Dr. David Benavente, llegando en 1923 a ser designado prosector. Llegó a Concepción en 1924 para realizar un año de internado obligatorio en el Hospital de Concepción. Ese mismo año fue designado por el Dr. González, como jefe de trabajos prácticos, labor que realizó en paralelo a la de profesor interino de la cátedra de anatomía para Dentística. Rápidamente su protagonismo fue notorio; en 1927 se encargó de la cátedra de anatomía para Medicina. En 1929 fue nombrado Director del naciente Instituto y posterior Departamento de Anatomía (Tabla I). Durante sus 38 años de labor docente logró que la anatomía de Concepción elevara su nivel, destacó la construccion del edificio del Departamento de Anatomía Normal y la creación del Museo de Anatomía Normal y Medicina Legal. Adicionalmente desarrolló labores de Decano de la Facultad de Medicina durante dos periodos, 1941- 1942 y 1943-1944. Su labor en el área de investigación también fue importante; entre sus trabajos destacaron: Variaciones de la columna vertebral entre chilenos (1940); Observaciones del orificio aórtico del diafragma (1942), Anastomosis de los nervios intercostales (1944); Contribución al estudio de las venas del simpático (1944); Contribución al estudio a las variaciones del plexo solar (1944), Consideraciones sobre un caso de fusión atlantooccipital (1952) y Técnicas para la preparación de linfáticos (1955), entre otros (Cicarelli, 1964). A lo anterior sumó la organización de congresos y eventos de difusión científica. Se acogió a jubilación en septiembre de 1961 y falleció en Santiago el 12 de julio de 1967. (Solervicens; Muñoz, 1993; Archivos del Departamento de Anatomía Normal y Medicina Legal Universidad de Concepción).

Edificio de Anatomía, Casa Definitiva y Consolidación de la Actividad Anatómica. Entrando a la década del 30, el Rector Sr. Enrique Molina comenzó a concretar el ansiado proyecto de un Campus Universitario coronado con un campanil. Este proyecto de ciudad universitaria se llevó a cabo en los terrenos del predio La Toma. El edificio destinado a las actividades de anatomía reemplazó el Pabellón Veneciano y fue el tercero en ser construido. Previamente fueron terminados los edificios de la Escuela Dental y el de Química Industrial. La construcción del pabellón definitivo fue encargado a los arquitectos Carlos Miranda y Enrique San Martín quienes siguieron los lineamientos conocidos como "Plan Bruner" (Muñoz, 1993; García). La arquitectura de este edificio siguió una línea marcada por un estilo ecléctico con claras influencias del Art Deco (Fig. 4). Su
Tabla I. Directores Departamento de Anatomía Normal y Medicina Legal, Universidad de Concepción, Chile.

\begin{tabular}{ll}
\hline Profesor & Año \\
\hline Dr. Enrique Solervicens Castel & $1929-1961 *$ \\
Dr. Edgardo Enríquez Fröden & $1961-1969$ \\
Dr. Mario Echeverría Monardes & $1970-1974$ \\
Dr. Eduardo Bustos Valdés & $1974-1982 / 1990-1992 * *$ \\
Dra. Nidia Epelde-Aguirre & $1982-1990$ \\
Dr. Luis Vielma Bustamante & $1992-1998$ \\
Dr. Hernán Tapia Rubilar & $1998-2002$ \\
Dr. Horacio Osorio Urbina & $2002-$ a la fecha \\
\hline
\end{tabular}

* El Departamento de Anatomia se crea en 1958.

** El Departamento cambia su denominación a Departamento de Anatomía Normal y Medicina Legal en 1990.

construcción se inició en 1932 y fue inaugurado a finales de 1933. Ha soportado múltiples terremotos, en el primero ellos, en 1939 que devastó la ciudad, tuvo que ser utilizado como Hospital de emergencia. Soportó sin grandes inconvenientes el terremoto de 1960, el cual provocó un incendio y posterior demolición del edificio de la Escuela Dental. El último de los terremotos, en 2010 provocó daños menores que fueron reparados ese mismo año. Entre 2015 y 2017 fue sometido a un proyecto de restauración que permitió modernizar los sistemas de manutención de cadáveres. Asimismo se reformaron sus salas de clases y laboratorios. Lo anterior permitió que el 13 de febrero de 2017 se publicara en el Diario Oficial el decreto mediante el cual el Consejo de Monumentos Nacionales declaró Monumento Nacional en la categoría Monumento Histórico a la zona de las primeras edificaciones del Campus de la Universidad de Concepción, zona que involucra al edificio de anatomía (Diario Oficial de la República de Chile, 2017). El Dr. Solervicens lo describió de la siguiente forma: "Las instalaciones de este nuevo local ofrecía varias ventajas, no solo por ser calefaccionado, sino que permitió mejorar notablemente las condiciones higiénicas de los trabajos de disección tanto por el aseo más cuidadoso como por la previa fijación del material cadavérico y la consiguiente superación de infecciones y malos olores".

Respecto a la consolidación de la actividad anatómica, esta se basó en tres aspectos: la dedicación exclusiva del Director del Departamento, la diversificación de técnicas para la preparación del material cadavérico y el inicio de la carrera académica al interior de la unidad. Al respecto, el Dr. Solervicens desde 1929 se desempeñó con dedicación exclusiva en el Instituto de Anatomía lo que le permitió estudiar y practicar un sinnúmero de técnicas anatómicas, muestra de ello es la adquisición de lupas para una mejor disección y el desarrollo de métodos de conservación cadavéricas como la diafanización y la repleción-corrosión, entre otras. Todo lo anterior impactó positivamente en la calidad de la docencia y de la incipiente investigación. Asimismo, desde 1947, los 

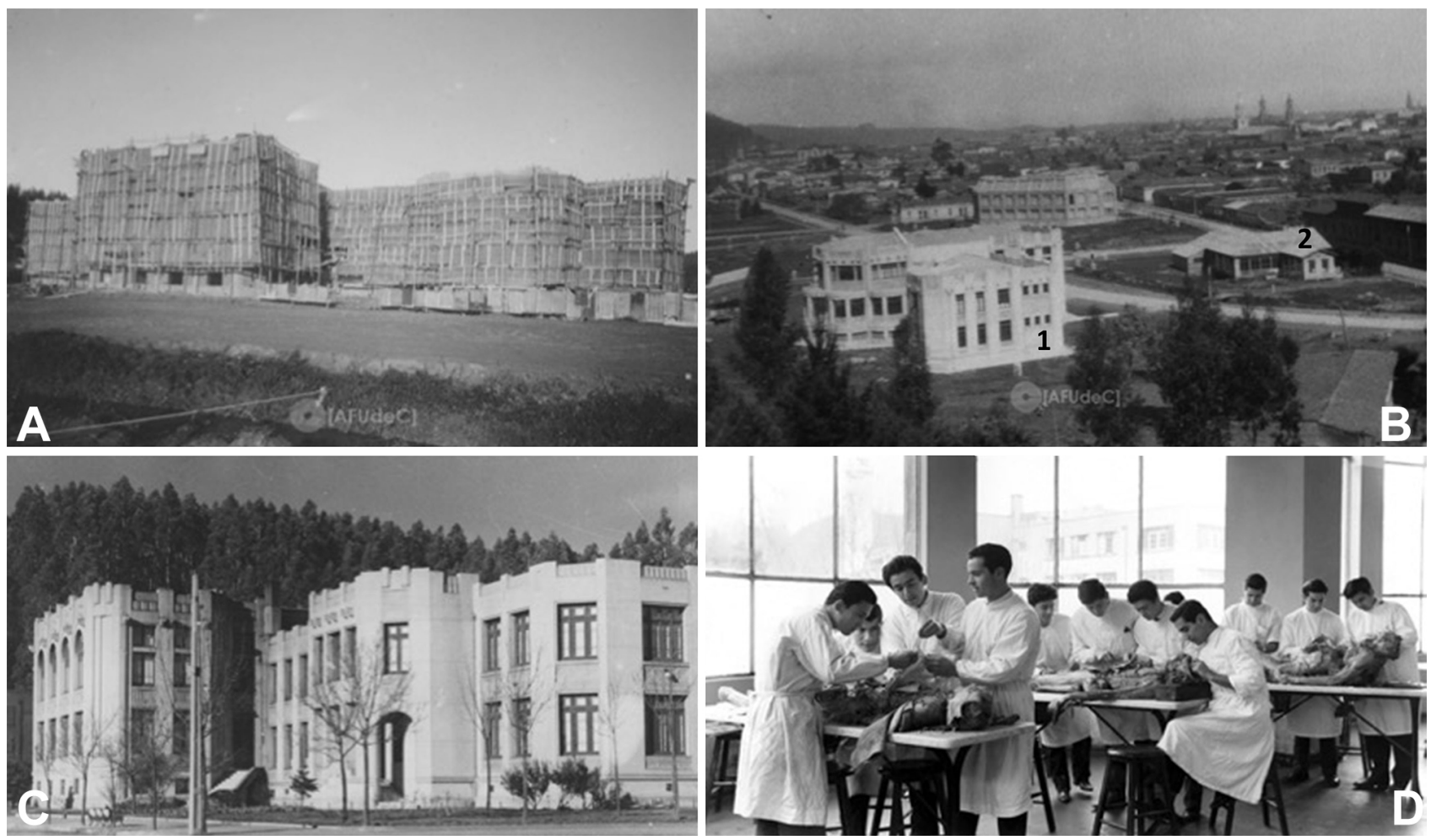

Fig. 4. Edificio de Anatomía, Barrio Universitario, Universidad de Concepción. A: Construcción del Edificio en 1932, B: Emplazamiento del Edificio (1: Edificio de Anatomía, 2: Pabellón Veneciano), C: Edificio entregado en 1933, D: Actividad práctica en el pabellón de disección (Archivo fotográfico Universidad de Concepción AFUdeC).

ayudantes alumnos fueron sustituidos por ayudantes profesionales (médicos cirujanos y odontólogos), los que tuvieron dedicación exclusiva; ello propició la generación de un semillero fructífero de profesionales e investigadores. De esos ayudantes profesionales en las décadas siguientes destacaron los Dres. Edgardo Enríquez Fröden y Mario Echeverria Monardes (Solervicens).

En lo que respecta a la modernización de los cursos de anatomía, en el caso de los estudiantes de medicina y odontología, desde 1936 se estableció una extensión de los trabajos prácticos a todo el cadáver, lo que se tradujo en que cada cuatro estudiantes se debían organizar para disecar un cadáver completo (Solervicens). Reflejo de esta modernización es la diversificación de los recursos para la enseñanza de la anatomía, entre las cuales destacaron la utilización de láminas anatómicas realizadas por los propios docentes o ayudantes, tal como sucedía en otros centros de estudio; más de un centenar de prepararados cadavéricos y dibujos esquemáticos en el pizarrón, entre otros (Fig. 5) (Cárdenas et al., 2017; Cárdenas-Valenzuela, 2019).

Acogido a jubilación y después de 38 años de dedicación anatómica, en 1961 el Dr. Solervicens dejó el Departamento de Anatomía en manos del Dr. Edgardo Enríquez Fröden quien ejerció labores hasta 1969. Durante su dirección se inició el curso de anatomía para la escuela de Obstetricia. En lo consecutivo, seis profesores han desempeñado la labor de Dirección del Departamento de Anatomía Normal y Medicina Legal (Tabla I). Otro reflejo de la intensa actividad académica es que esta casa de estudios ha sido el organizador del V Encuentro Nacional de Anatomía, en 1983, el XI Encuentro Nacional de Anatomía, en 1989 y el XVII Congreso Chileno de Anatomía, en 1996 (CárdenasValenzuela \& Rodríguez-Torres, 2018).

** El Dr. Edgardo Enríquez Fröden (Fig. 1D) es uno de los penquistas más influyentes de la segunda mitad del siglo XX. Nació en Concepción el 9 de febrero de 1912. Estudió en el Liceo de Hombres de Concepción siendo el mejor alumno de su promoción. Posteriormente, entre los años 1930 y 1936, estudió medicina en la Universidad de Concepción. En el transcurso de sus estudios universitarios obtuvo el premio Arturo L. de Ambroccy por ser el mejor estudiante de su generación. En 1936 inició sus actividades académicas como ayudante alumno del Instituto de Anatomía de la Universidad de Concepción. Tres años más tarde fue designado Jefe de trabajos prácticos. Ese mismo año comenzó su carrera en la Armada de Chile como Ooficial de Sanidad, de la cual se acogió a retiro 30 años más tarde con el grado de Capitán de Navío. Durante su vida profesional en la Armada también desempeñó el cargo de Director del Hospital Naval "Almirante Adriazola" de la Base Naval de Talcahuano.

Entre los años 1955 y 1956 y producto de haber obtenido una la beca de la Kellogg Foundation, siguió cursos de perfeccionamiento en anatomía humana dictados por el profesor Charles Mayo Goss de la Universidad de Loussiana State, Estados Unidos. De regreso al país, en la 


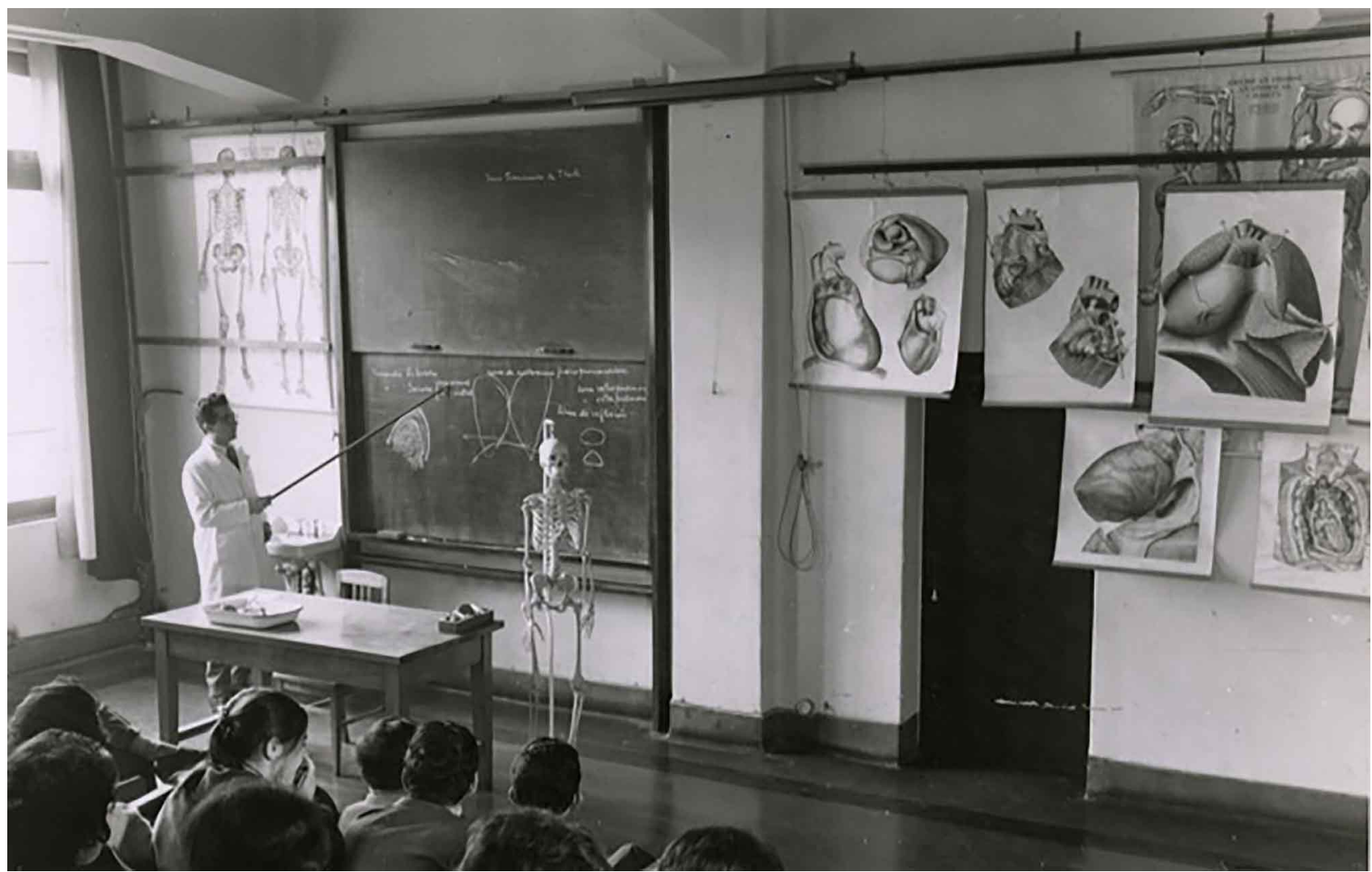

Fig. 5. Sala Virgínio Gómez. Clase de Anatomía cardiaca en la década del 60 a cargo del Dr. Castillo. (Archivo fotográfico Universidad de Concepción AFUdeC).

Universidad de Concepción ocupó numerosos cargos relevantes; fue profesor de anatomía y Director del Departamento de la misma entre los años 1961 y 1969. En el año 1969, en plena reforma universitaria fue elegido Rector, cargo que ejerció hasta 1972. En el año 1973 fue nombrado Ministro de Educación por el presidente Salvador Allende Gossens lo que le valió estar el año 1974 detenido la isla Dawson y posteriormente enviado al exilio. Pasó primero por Inglaterra para después radicarse en México. En 1979 ejerció como profesor de anatomía en la Universidad Autónoma Metropolitana de Xochimilco. En 1990 fue reincorporado a la Escuela de Medicina de la Universidad de Concepción, siendo nombrado en 1991 Profesor Emérito. En 1980 publicó el texto "Anatomía del sistema nervioso central" para después escribir sus memorias denominadas "En el nombre de una vida". A los 83 años, falleció el 1 de noviembre de 1996. (Solervicens; Archivos del Departamento de Anatomía normal y Medicina Legal Universidad de Concepción).

Museo de Anatomía. Legado morfológico. Patrimonio Científico de Chile, el Museo de Anatomía de la Universidad de Concepción fue fundado en la década de 1930 por el Dr. Solervicens para ayudar en la enseñanza, investigación y formación de los profesionales de la salud. Para ello contó con la valiosa colaboración de su discípulo Mario Echeverría. Hoy, el Museo de Anatomía es visitado anualmente por mas de 2.500 personas, siendo un atractivo penquista en el día del Patrimonio Cultural. Ubicado en una amplia sala en el interior del edificio de anatomía, este museo reune en diferentes vitrinas los principales órganos, sistemas y regiones topográficas que conforman el cuerpo humano. Abordan preparados cadavéricos en adultos, niños y fetos. Entre sus vitrinas destacan preparados con la técnica de replecióncorrosión, la cual fue aplicada en la distribución traqueobronquial, distribución vascular hepática y coronaria. Otros de los puntos de interés es la sección de medicina forense en donde se evidencian las lesiones generadas por traumatismos directos, balísticos o quemaduras, ya sean por fuego o por electricidad (Facultad de Medicina Universidad de Concepción, 2019).

** El Dr. Mario Echeverría es uno de los grandes exponentes del apogeo de la anatomía. Nació en Chillán el 18 de septiembre de 1926, allí estudió en su Liceo de Hombres. En 1951 se graduó de Cirujano Dentista de la Universidad de Concepción, complementariamente, en 1977 se tituló de Médico Cirujano. En 1951 inició su carrera académica en la Universidad Concepción como ayudante de Clínica Operatoria. Al año siguiente ingresó con dedicación exclusiva como ayudante profesional al Departamento de Anatomía. Entre 1958 y 1959 y producto de una beca concedida por el Gobierno Francés, estudió en la Université de Lyon donde trabajó con Michael Latarjet. A lo anterior se sumó una estadía en Amsterdam 1965-1966 y otra en el laboratorio de anatomía de la Faculté de Médecine de la Université de París V, con el profesor André Delmás (1966-1968 y 1978-1980). Dicha casa de estudios lo designó Assistant Etranger y Attache-Assistant de Sciencies Fondamentales; adicionalmente recibió de la Faculté de Médecine de la Université Pierre y María Curie en conjunto con L'Hôpital Pitie-Salpétriere, una certificación como especialista en neurología. Los frutos de su desarrollo académico en Francia le valieron en 1981 recibir el premio "Laureat De L'Academie Nationale". 
A pesar de sus viajes por perfeccionamiento, su vínculo con la Universidad de Concepción fue permanene, allí dictó la cátedra de anatomía para las Escuelas de Medicina y Odontología. Entre 1970 y 1974 fue Director del Departamento de Anatomía. Su extensa contribución académica se refleja en más de 50 preparados cadavéricos que forman parte del Museo de Anatomía de esta casa de estudios. A lo anterior se agregan 20 láminas anatómicas pintadas en acuarela y múltiples investigaciones publicadas, varias de ellas con su maestro el Dr. Solervicens. En 1977 retorna a Francia para tratarse una enfermedad originada por la exposición a la formalina. Se acogió a jubilación el año 1996 y falleció el año 2008 (Archivos del Departamento de Anatomía Normal y Medicina Legal Universidad de Concepción).

La extensa actividad anatómica de la Universidad de Concepción cumplió 100 años desde su inicio, en ella han participado más de un centenar de profesores que han formado miles de profesionales que contribuyen al país. De este largo listado destacaron los cinco pioneros que han sobresalido entre sus pares, sin embargo la contribución de todos sus profesores, ayudantes y laborantes es invaluable.

Actualmente la enseñanza de la anatomía es una realidad en las ocho universidades de la Región del BioBio; de ellas; las Universidades San Sebastián, Andrés Bello y de las Américas tiene departamentalizada la actividad anatómica.

A pesar de transcurridos un siglo de actividad anatómica, las frases del celebre médico Dr. Lucas Sierra escritas en 1924 siguen válidas: “..... Nos parece asunto ineludible y de la mayor trascendencia que el profesor de anatomía, convencido de que en la ciencia pura nada hay que no sea o puede ser de gran importancia, sepa insistir ante sus alumnos, estudiantes solamente de medicina, en los hechos de real y positiva utilidad para el médico práctico; que no caiga en la tentación de hacerle aprender hasta las más insignificantes rugosidades de un hueso o las innumerables anomalías que puedo ofrecer en su inserción este o aquel musculillo; eso no es más que "pedantería anatómica" y no contribuye si no a distanciar el gusto por el estudio de la anatomía, que hara bien en conservar su vida entera todo médico digno de su nombre.... enseñemos entonces la ANATOMÍA VIVA" (Sierra, 1924).

AGRADECIMIENTOS. Agradecemos al Archivo Fotográfico de la Universidad de Concepción por su contribución gráfica a esta comunicación científica.

OSORIO, H.; TORO, J.C.; SCHORWER, K.; RIVEROS, A. \& CARDENAS, J. Pioneers of a Century of Anatomical Teaching in the City of Concepción, Chile. Int. J. Morphol., 38(3):650-658, 2020.

SUMMARY: Reviewing a century of history, the development of anatomical activities in the city of Concepción, in the south of Chile, did not occur without difficulties. The present work gathered the historical background related with the beginnings and development of Anatomy teaching at the Universidad de Concepcion. Research was carried out including the review of historical books, magazines, digital archives and institutional records such as files or photographic archives. The foundation of the university, the beginnings of anatomical activities in 1919 and the contribution of its highlighted pioneers, show the difficulties of teaching this discipline in Chile. From a historical perspective, having knowledge during this century of teaching, the physical resources used in cadaveric dissection, the detail of teaching methodologies applied, and the anatomical resources available, contribute the anatomy's development knowledge in Chile.

KEY WORDS: Anatomy: Concepción; History of Anatomy; Teaching of Anatomy; National Monument.

\section{REFERENCIAS BIBLIOGRÁFICAS}

Archivos del Departamento de Anatomía Normal y Medicina Legal Universidad de Concepción, años 1960 a 2005. Concepción. Universidad de Concepción, 2019.

Cárdenas-Valenzuela, J \& Rodríguez-Torres, A. Chilean Society of Anatomy. The beginning. Int. J. Morphol., 36(3):1075-8, 2018.

Cárdenas-Valenzuela, J. Teaching of anatomy. Use of media in the classroom. Int. J. Morphol., 37(3):1123-9, 2019.

Cárdenas, V. J. L.; Dinator, E. M. \& Madariaga, L. J. Description of the anatomical plates of the Universidad de Chile, a national monument. Int. J. Morphol., 35(2):711$8,2017$.

Cavieres, F. E. Los jesuitas expulsos: La comunidad y los individuos. La provincia de Chile. Cuad. Hist., (38):7-38, 2013.

Cicarelli, A. N. Apuntes para una bibliografía chilena de anatomía. An. Chil. Hist. Med 6(2):88-112, 1964.

Da Costa, M. Crónica Fundacional de la Universidad de Concepción (1917-1920). Concepción, Universidad de Concepción, 1995

Da Costa, M. La Universidad de Concepción. Concepción Vivir su Historia. Concepción. Ediciones Sociedad de Historia de Concepción, 2000.

Diario Oficial de la República de Chile, Decreto Número 393. Declara Monumento Nacional en la categoría de Monumento Histórico al "Campus central de la Universidad de Concepción”, ubicado en la comuna y provincia de concepción, región del Biobío. Diario Oficial de la República de Chile, N 41.683, 13 de febrero de 2017.

Facultad de Medicina Universidad de Concepción. Sitio Web. Concepción, Facultad de Medicina Universidad de Concepción, 2019. Disponible en: www.udecmed.cl/ facultad/historia/

García, J. El Campus de la Universidad de Concepción. Su Desarrollo Urbanístico y Arquitectónico. Concepción, Universidad de Concepción, 1994.

Jaramillo, E. Memoria del Director de la Escuela de Medicina. Rev. Medde Chile, 52:714, 1924.

Luvel, R. Crónicas y Semblanzas de Concepción. Concepción, Ilustre Municipalidad de Concepción, 1968.

Muñoz, C. Historia de la Universidad de Concepción. Concepción, Facultad de Medicina Universidad de Concepción, 1993.

Muñoz, C. Virginio Gómez Gonzalez. Visionario y Fundador. Concepción, Trama impresores, Corporación Educacional Masónica de Concepción, 2015.

Sierra. L. La Universidad de Concepción y la enseñanza de la anatomía viva. Atenea, 1(3):176-9, 1924.

Solervicens, E. Desarrollo de la anatomía en Concepción. An. Chil. Hist. Med., 6(2):7687, 1964.

Wilhelm, O. Historia de la fundación de la escuela de medicina de la Universidad de Concepción. An. Chil. Hist. Med., 8(1):89-112, 1966.

Wilhelm, O. Historia de la medicina penquista. An. Chil. Hist. Med., 4(1):103-30, 1962.

Dirección para Correspondencia:

Klgo. Mg. Andrés Riveros Valdés.

Depto. de Ciencias Morfológicas

Universidad San Sebastián

Sede Concepción

Lientur 1457 C.P: 4080871

Concepción - CHILE

Recibido : 27-11-2020

Aceptado: 03-01-2020

Email: andres.riveros@uss.cl 